\title{
The Role and Mechanism of CRT0066101 as an Effective Drug for Treatment of Triple- Negative Breast Cancer
}

\author{
Yan Liua, ${ }^{a, b, c, d}$ Yuzhi Wang ${ }^{b, d} \quad$ Shiyi Yub,d Yehui Zhoue Xinxing Mae \\ Qian Su $^{\mathrm{a}}$ Li An ${ }^{\mathrm{a}} \quad$ Feifei Wang $^{\mathrm{a}} \quad$ Aihua Shi $^{\mathrm{a}}$ Jingzhong Zhang ${ }^{\mathrm{a}}$ \\ Liming Chen ${ }^{\mathrm{b}, \mathrm{d}}$
}

\begin{abstract}
aThe Key Laboratory of Bio-Medical Diagnostics, Suzhou Institute of Biomedical Engineering and Technology, Chinese Academy of Sciences, Suzhou, China, bjiangsu Key Laboratory for Molecular and Medical Biotechnology, College of Life Science, Nanjing Normal University, Nanjing, China, 'Changchun Institute of Optics, Fine Mechanics and Physics, Chinese Academy of Sciences, Changchun, China, 'The Key Laboratory of Developmental Genes and Human Disease, Ministry of Education, Institute of

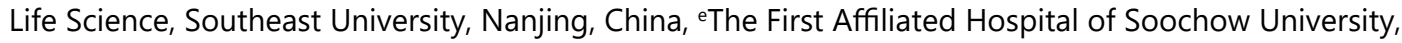
Soochow University, Suzhou, China
\end{abstract}

\section{Key Words}

Triple-negative breast cancer • CRT0066101 • PRKD • Phosphoproteome

\begin{abstract}
Background/Aims: Breast cancer is clinically classified into three main subtypes: estrogen receptor-positive $\left(E R^{+}\right)$breast cancer, human epidermal growth factor receptor 2-positive $\left(\mathrm{HER2} 2^{+}\right)$breast cancer, and triple-negative breast cancer (TNBC). Without specific targeted therapies, patients with TNBC have poorer prognosis compared with those with $\mathrm{ER}^{+}$and $\mathrm{HER} 2^{+}$ breast cancer. Protein kinase D (PRKD) family members play crucial roles in cancer progression. CRT0066101, a PRKD inhibitor, has been reported to have anticancer activity in many cancer types. Nevertheless, the role and mechanism of CRT0066101 in TNBC have not been well investigated. Methods: The expression level of PRKDs was analyzed in breast cancer samples and breast cancer cell lines. The effects of inhibiting PRKD activity with CRT0066101 on TNBC cell proliferation, cell cycle, apoptosis, and tumor growth were studied by Cell Counting Kit8 assay, cell cycle assay, propidium iodide/annexin- $V$ assay, and a xenograft mouse model, respectively. To uncover the molecular mechanism of CRT0066101 in TNBC, comparative phosphoproteomic analysis using iTRAQ was employed. Results: We found that PRKD2 and PRKD3 were preferentially expressed in breast cancers. Immunohistochemistry confirmed the overexpression of PRKD2 and PRKD3 in TNBC. CRT0066101, which inhibited the activity of PRKDs, dramatically inhibited proliferation, increased apoptosis and the G1-phase population of TNBC cells in vitro, and reduced breast tumor volume in vivo. Comparative phosphoproteomic
\end{abstract}


analysis between breast cancer cells with and without CRT0066101 treatment revealed that the anti-breast cancer effects involved regulation of a complex network containing multiple enriched pathways and several hub-nodes contributing to multiple cancer-related processes, thus explaining the described effects of CRT0066101 on TNBC in vitro and in vivo. Finally, we validated several targets of PRKD inhibition by treatment with CRT0066101 and small interfering RNAs against PRKD2 and PRKD3 (siPRKD2 and siPRKD3), including p-MYC(T58/ S62), p-MAPK1/3(T202/Y204), p-AKT(S473), p-YAP(S127), and p-CDC2(T14). Conclusion: PRKD inhibitor CRT0066101 exhibits anti-TNBC effects via modulating a phosphor-signaling network and inhibiting the phosphorylation of many cancer-driving factors, including MYC, MAPK1/3, AKT, YAP, and CDC2, providing insight into the important roles as well as the molecular mechanism of CRT0066101 as an effective drug for TNBC.

(C) 2019 The Author(s). Published by Cell Physiol Biochem Press GmbH\&Co. KG

\section{Introduction}

Breast cancer, the most common type of cancer in women, is clinically classified into estrogen receptor-positive $\left(\mathrm{ER}^{+}\right)$breast cancer, human epidermal growth factor receptor 2-positive $\left(\mathrm{HER}^{+}\right)$breast cancer, and triple-negative breast cancer (TNBC). TNBC is characterized as $\mathrm{ER}^{-}$, progesterone receptor-negative, and HER2-. Owing to the lack of a molecular target, cytotoxic radiotherapy and chemotherapy are the only choices for TNBC patients [1]. However, the recurrence rate is high after cytotoxic chemotherapy and radiotherapy [2]. TNBC's high resistance to therapy has been attributed to tumors showing mesenchymal and stem cell features, and there are no specific targeted therapies [3-5]. TNBC is resistant to standard hormone therapy and HER2-directed agents such as trastuzumab, due to the loss of expression of hormone receptors and HER2 [6, 7]. The present methods for treating TNBC mainly consist of surgical resection, auxiliary assist, and localized chemotherapy, but postoperative recurrence is difficult to control. Thus, development of new therapeutic drugs for TNBC is important.

The protein kinase D (PRKD) family consists of serine/threonine kinases that belong to the calcium/calmodulin-dependent kinase (CaMK) superfamily. It contains three members: PRKD1, PRKD2, and PRKD3. PRKDs have been reported in multiple cancer-promoting processes such as cell proliferation [8, 9], cell migration [10,11], epithelial-mesenchymal transition [12], and angiogenesis [13]. Differences in function between PRKD isoforms have been described in breast, colon, gastric, and prostate cancers [10, 14-20]. In breast cancer, PRKD1 has been shown to promote cell growth [21] and inhibit invasiveness [22, 23]. By comparison, PRKD2 and PRKD3 promote proliferation, metastasis, and chemoresistance in breast cancer progression [14, 24, 25]. In TNBC, PRKD1 is silenced by hypermethylation [26]. In contrast to PRKD1, PRKD2 and PRKD3 have been found by our and other research groups to play important roles in promoting oncogenic transformation of TNBC $[14,24,27$, 28]. Until now, several small-molecule inhibitors have been shown to inhibit PRKD activity in vitro, such as CID755673 and its analogs [15, 29], 3, 5-diarylazoles [30], 2, 6-naphthyridine and bipyridyl inhibitors and their analogs [31], CRT5 [32], and CRT0066101 [33]. These compounds effectively block proliferation, migration, and invasion in vitro [34]. CRT0066101, a small-molecule PRKD inhibitor, was reported to show potential anticancer effects in xenograft models of pancreatic [33], colorectal [35], and breast cancer [27]. According to these reports, CRT0066101 was well tolerated and had no significant side effects in mice, therefore suggesting CRT0066101 as a promising agent for clinical trials. However, a thorough understanding of the anticancer effects and underlying mechanism of CRT0066101 in TNBC is needed for further validation and exploration to facilitate its clinical use.

A more comprehensive understanding of the identity of the candidate phosphoproteins affected by PRKD inhibition by CRT0066101 will be important to understand the role of PRKDs and CRT0066101 in cancer. We therefore used iTRAQ comparative phosphoproteomic 
analysis to uncover global effects of CRT0066101 in TNBC. The results provide new insight into the functions of PRKDs and clues for combating TNBC with CRT0066101.

\section{Materials and Methods}

\section{Breast cancer samples}

We obtained all the breast cancer samples from Nanjing General Hospital. After resection, samples were immediately cryopreserved in liquid nitrogen. Hematoxylin and eosin-stained frozen sections were prepared from each mammary gland specimen to confirm the diagnosis and to obtain histopathological classifications and molecular subtypes. All the breast cancer specimens were analyzed anonymously after obtaining the patients' written consent. This study was approved by the medical ethics committee of Suzhou Institute of Biomedical Engineering and Technology.

\section{Cell culture}

MCF7, MDA-MB-468, BT549, and MDA-MB-231 cells were maintained in Dulbecco's modified Eagle's medium (Life Technologies) supplemented with $1 \%$ penicillin-streptomycin solution (Life Technologies) and 10\% fetal bovine serum (HyClone). T47D and HS578T cells were cultured in RPMI 1640 medium (Corning Cellgro) supplemented with 1\% penicillin-streptomycin solution and 10\% fetal bovine serum. All the cell lines used in this study were from the American Type Culture Collection and used within 6 months.

\section{Western blot}

For western blot analysis, primary antibodies against PRKD1, PRKD2, PRKD3, p-PRKD(Ser744/748), AKT, p-AKT(S473), YAP, p-YAP(S127), MAPK1/3, p-MAPK1/3(T202/Y204), CDC2, MYC, and p-MYC(S62) were purchased from Cell Signaling Technology. p-CDC2(T14) antibody was purchased from Signalway Antibody LLC. p- MYC(T58) antibody was purchased from ABM. Anti-rabbit secondary antibody, anti-mouse secondary antibody, and $\beta$-actin primary antibody were purchased from Santa Cruz Biotechnology. Western blotting was carried out following standard protocols.

\section{Cell proliferation, cell cycle, and cell apoptosis assays}

Cell proliferation was determined by using Cell Counting Kit-8 reagent (Dojindo Laboratories) according to the manufacturer's instructions. For cell cycle analysis, unsynchronized cells were digested with trypsin and fixed with 70\% ethanol. DNA was stained with propidium iodide and the cell cycle distribution was analyzed by flow cytometry using a BD FACSCalibur (Becton Dickinson). For the cellular apoptosis experiments, an Annexin-V/Dead Cell Apoptosis Kit (Invitrogen) was used to stain the cells according to the manufacturer's instructions and analyzed on the BD FACSCalibur flow cytometer.

\section{In vivo mouse model}

Female athymic STOCK-Foxn1 ${ }^{\text {nu}} / \mathrm{Nju}$ 4-week-old mice were obtained from Shanghai Sushang Biology Technology Co., Ltd. Breast cancer cells $\left(5 \times 10^{6}\right.$ cells $)$ were subcutaneously injected into the armpit of mice. Upon CRT0066101 treatment, nude mice bearing MDA-MB-231 and MDA-MB-468 tumor xenografts were administered daily vehicle control (dextrose) or CRT0066101 at $80 \mathrm{mg} / \mathrm{kg}$ orally. A Vernier caliper was used to calculate tumor volume by the following formula: volume $=0.5 \times$ length $\times$ width $^{2}$. All of the experiments were conducted in accordance with the instructional standard guidelines of Suzhou Institute of Biomedical Engineering and Technology for animal experiments.

\section{PRKD-regulated phosphoproteomic analysis using iTRAQ}

Cells were prepared using a ReadyPrep Protein Extraction kit (Bio-Rad Laboratories). Extracted protein concentration was determined by a BioSpec-nano (Shimadzu Biotech, Kyoto, Japan). Approximately $4 \mathrm{mg}$ of protein/sample was used for quantitative phosphoproteomic profiling. Each protein sample was reduced and alkylated and digested with trypsin (Promega). Following tryptic digestion, peptide samples were desalted using a MonoTip C18 (Shimadzu Biotech). The eluted peptides were dried in a SpeedVac and then labeled with iTRAQ 8-plex reagents according to the manufacturer's instructions. 


\title{
Cellular Physiology Cell Physiol Biochem 2019;52:382-396 \\ \begin{tabular}{ll|l} 
and Biochemistry & $\begin{array}{l}\text { DOl: 10.33594/000000027 } \\
\text { Published online: } 5 \text { March 2019 }\end{array}$ & $\begin{array}{l}\text { O } 2019 \text { The Author(s). Published by } \\
\text { Cell Physiol Biochem Press GmbH\&Co. KG }\end{array}$ \\
\cline { 2 - 3 }
\end{tabular}
}

Liu et al.: Quantitative Phosphoproteomics Reveals Mechanism of CRT0066101

\begin{abstract}
Phosphopeptide enrichment was performed using a Titansphere Phos-TiO Kit (Shimadzu Biotech) according to the manufacturer's instructions. Eluted phosphopeptides were combined, acidified with $100 \mathrm{~mL}$ of $2.5 \%$ trifluoroacetic acid, desalted with the MonoTip C18, and resuspended in $0.1 \%$ formic acid. Samples were analyzed using a Prominence nanoflow liquid chromatography system (Shimadzu Biotech) connected to a liquid chromatography-mass spectrometry ion-trap time-of-flight mass spectrometer (Shimadzu Biotech). The detected fragments were analyzed with ProteinLayer software using the Swiss-Prot human database and the phosphorylation sites were determined using PTM Finder Software (Shimadzu Biotech).

Abundance ratios between samples were quantified by LabSolution Software (Shimadzu Biotech) via the quantification of iTRAQ-labeled peptides, with a synthetic peptide corresponding to the residues between 14 and 38 (TQCPDDSTCCELPTGK) of mouse granulin-3 labeled with [d0]/[d6]-DMPITC used as an internal standard for quantification (BioworldInc, Minneapolis, MN). To minimize contamination from isobaric ions, only the peptides with isolation specificity greater than $75 \%$ were quantified.

Statistical analysis

GraphPad Prism version 5.0 software was used for all statistical analyses. Data were expressed as the mean \pm standard error of the mean (SEM).
\end{abstract}

\section{Results}

PRKD2 and PRKD3 are the two major PRKDs expressed in breast cancer

To test the role of PRKDs in breast cancer, we first examined the gene expression data for 1888 breast cancer samples from The Cancer Genome Atlas (TCGA) and found that PRKD2 and PRKD3 were the two major PRKD isoforms expressed relative to PRKD1 (Fig. 1A and Supplementary Table 1 - all supplementary material available online at www.cellphysiolbiochem.com). Second, we explored the PRKD1, PRKD2, and PRKD3 gene expression data in breast cancer cell lines [36] and found that PRKD2 and PRKD3 were the two major isoforms expressed among all PRKDs in TNBC cell lines (Fig. 1B and Supplementary Table 2). Third, we collected 34 breast tumor samples and verified that PRKD2 and PRKD3 expression was higher than PRKD1 according to quantitative reverse transcription PCR (RT-qPCR) (Fig. 1C and Supplementary Table 3). Fourth, analysis of TNBC by immunohistochemistry also confirmed that PRKD2 and PRKD3 levels were elevated in TNBC tissues compared with precancerous tissues (Fig. 1D). To select suitable cell models, we carried out western blotting on the breast cancer cell lines and selected MDA-MB-231 and MDA-MB-468, which express PRKD2 and PRKD3 and not PRKD1, as model TNBC cell lines (Fig. 1E).

Anti-TNBC effects of PRKD inhibitor CRT0066101 in vivo and in vitro

To test the anti-TNBC effects of CRT0066101, we first carried out in vitro assays, including proliferation, cell cycle, and apoptosis assays. The results show that CRT0066101 induced a marked reduction in proliferation in the investigated TNBC cell lines (Fig. 2A), an increase in the population of cells in G1 phase and a decrease in S-phase cells (Fig. 2B), and increased apoptosis (Fig. 2C), suggesting that CRT0066101 exhibited anti-TNBC effects in vitro. However, it did not have a discernible toxic effect on the noncancerous breast cell line MCF10A compared with PRKD2- or PRKD3-overexpressing breast cancer cell lines (Supplementary Fig. S1). To further validate the anti-TNBC effects of CRT0066101 using in vivo xenograft mouse models, we confirmed that CRT0066101 significantly reduced TNBC tumor volume in vivo (Fig. 3). Taken together, our results suggest that CRT0066101 is effective in the treatment of TNBC.

\section{Identification of phosphoproteomes after treating MDA-MB-231 with CRT0066101}

Since PRKDs are serine/threonine kinases, iTRAQ comparative phosphoproteomic analysis was used to reveal phosphorylation events affected by CTR0066101 in TNBC cells. Fig. 4A shows the workflow of analysis of MDA-MB-231 cells treated with phosphate- 


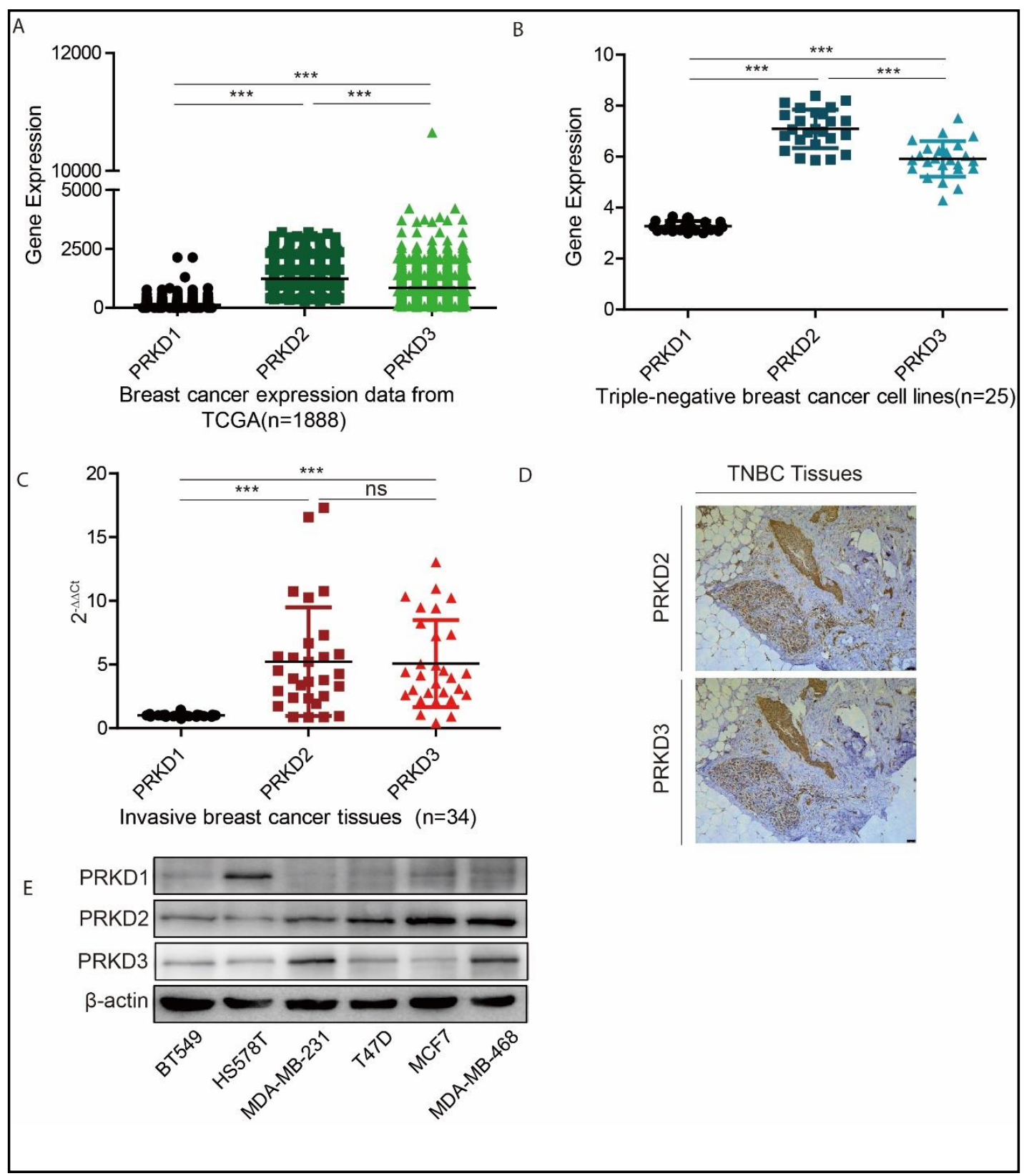

Fig. 1. PRKD expression in breast cancer. (A) PRKD expression in 1888 breast cancer tissues from the TCGA database. (B) PRKD expression in 25 TNBC cell lines. (C) PRKD expression in 34 collected breast cancer tissues by RT-qPCR. (D) Expression of PRKDs in TNBC tissues by immunohistochemistry. (E) PRKD expression in breast cell lines determined by western blot. Data represent the mean $\pm \mathrm{SEM}$. ${ }^{* * *} \mathrm{P}<0.001$ by t test.

buffered saline or CRT0066101. Each group contains two experimental duplicates. In total, 2252 phosphopeptides matching 3034 phosphosites from 1353 phosphoproteins were identified with a $P$ value $<0.005$ (Fig. 4B and Supplementary Table 4).

We regarded a phosphosite in a phosphopeptide of a particular phosphoprotein as being PRKD regulated if we detected a 1.5-fold or greater change between the sample and control. In total, 2232 phosphopeptides matching 3002 phosphosites from 1347 phosphoproteins were identified as being PRKD regulated (Fig. 4B and Supplementary Table 5). 


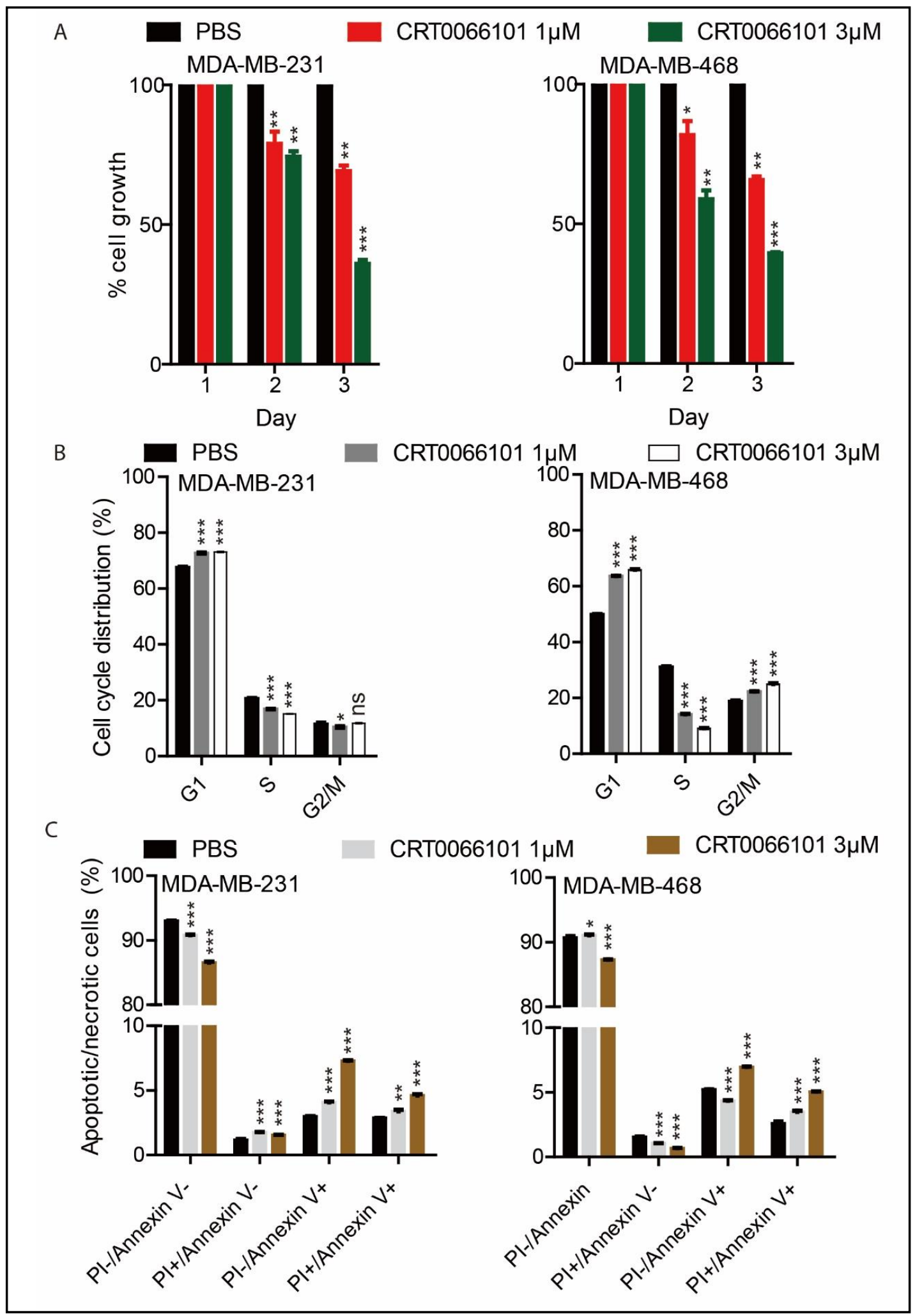

Fig. 2. CRT0066101 alters the biological behaviors of TNBC. TNBC cells were treated with 0,1 , and $3 \mu \mathrm{M}$ CRT0066101 and cell proliferation was determined via (A) Cell Counting Kit-8 assay, and (B) cell cycle and (C) cell apoptosis were analyzed by flow cytometry. Data represent the mean $\pm \mathrm{SEM}$. ${ }^{*} \mathrm{P}<0.05,{ }^{* *} \mathrm{P}<0.01$, and *** $\mathrm{P}<0.001$ by t test. 


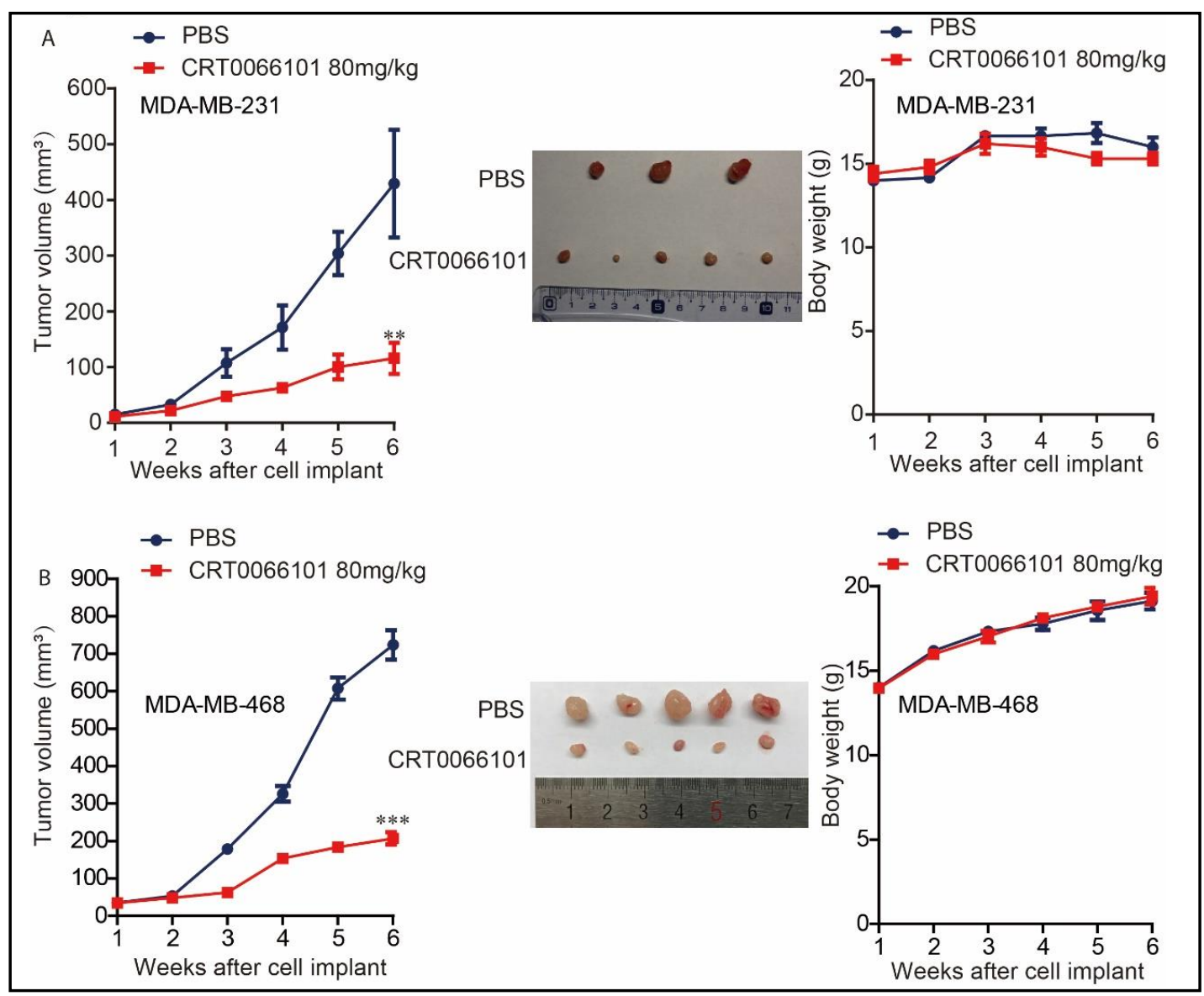

Fig. 3. CRT0066101 suppresses TNBC growth in vivo. CRT0066101 inhibited triple-negative breast tumor growth in a xenograft mouse model using (A) MDA-MB-231 and (B) MDA-MB-468 cells. Palpable tumor growth across time was measured every week from the time tumor was palpable until the animals were sacrificed (week 6). Tumor images at the end point (week 6) are shown and palpable tumor volumes were measured by width and length with a Vernier caliper and calculated using the formula volume $=$ (length $\times$ width $^{2}$ )/2. Data represent the mean \pm SEM. ${ }^{* *} \mathrm{P}<0.01$ and ${ }^{* * *} \mathrm{P}<0.001$ by $t$ test.

\section{Systematic analysis of CRT0066101-related phosphoproteome}

To further characterize the mechanism of CRT0066101 in TNBC, the derived comparative phosphoproteomic data first underwent reactome analysis [37]. The results show enriched reactomes involved in various biological events, such as RNA metabolism, cell cycle, gene expression, protein metabolism, disease, programmed cell death, immune system, and DNA repair (Supplementary Fig. S2). There were 52 pathways with $P<0.01$ and FDR $<0.01$ enriched in the reactomes of CRT0066101-related phosphoproteins (Supplementary Table 6). Among these 52 pathways, 12 are involved in RNA metabolism, 11 are involved in cell cycle, 8 are involved in protein metabolism, 7 are involved in disease, and 6 are involved in gene expression (Fig. 5A).

The networks of CRT0066101-related phosphoproteins were further investigated using the IMEx and STRING interactome databases. The nodes with the top 15 largest number of degrees or betweenness were suggested to be the hub-nodes in the networks. In the CRT0066101-related phosphoprotein network analyzed using the IMEx interactome database, the 15 nodes identified as hub-nodes were ELAVL1, SUMO2, UBC, HSP90AA1, MYC, CUL1, HSP90AB1, HDAC1, VCP, BRCA1, VHL, IKBKG, HDAC2, SRC, and CTNNB1 (Fig. 5B and Supplementary Table 7). In the CRT0066101-related phosphoprotein network analyzed 


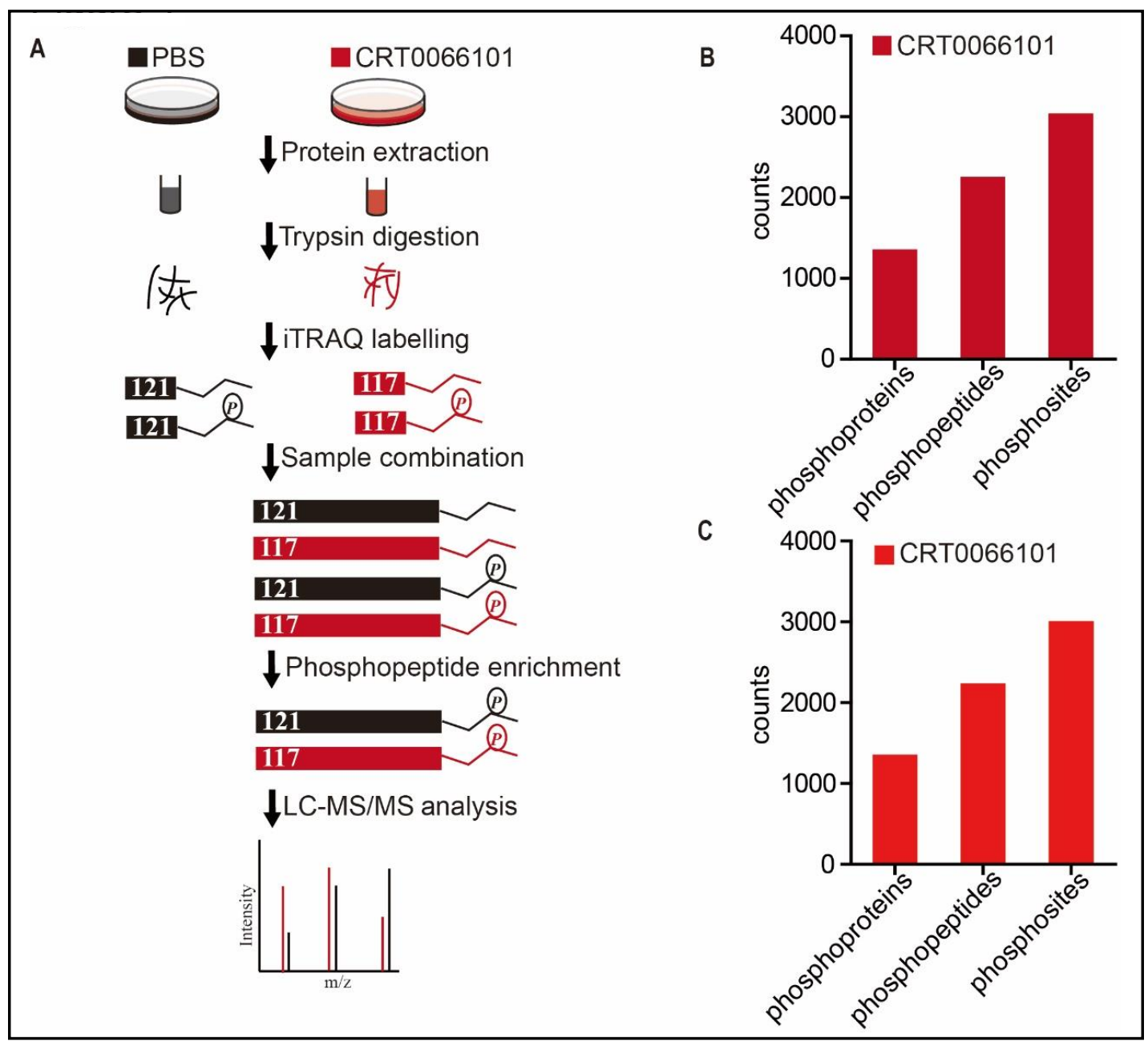

Fig. 4. Phosphoproteomic analysis of CRT0066101-treated MDA-MB-231 cells. (A) Flowchart of phosphoproteomic analysis. (B) Analysis of identified phosphoproteins, phosphopeptides, and phosphosites from the phosphoproteome. (C) Analysis of identified PRKD-regulated phosphoproteins, phosphopeptides, and phosphosites.

using the STRING interactome database, the 15 nodes identified as hub-nodes were UBC, SRC, HSP90AA1, HDAC1, CTNNB1, MAPK1, MAPK14, HDAC2, JUN, RPS14, SHP90AB1, MYC, MAPK3, and CUL1 (Fig. 5C and Supplementary Table 8).

CRT0066101 suppresses the phosphorylation of MYC, MAPK1/3, AKT, YAP, and CDC2

Since PRKD activity is suppressed by CRT0066101, it is plausible that the phosphorylation levels of downstream signaling proteins regulated by PRKDs decrease. To further explore the mechanism of CRT0066101 in breast cancer, we intended to validate several important targets of CRT0066101. Via integrating the result of NetworkAnalyst and PRKD-regulated phosphoproteins, we chose a series of significantly downregulated phosphoproteins as well as potential functionally important targets for further validation.

As described above, CRT0066101 inhibited proliferation, increased apoptosis, and increased the G1-phase population of TNBC cells. In the current study, MYC was found as a most important hub-node in PRKD-regulated pathways using the IMEx interactome database and STRING interactome database; MAPK1 and MAPK3 as CRT0066101-regulated phosphoproteins were two hub-nodes in the CRT0066101-related network. Although YAP, AKT, and CDC2 were not hub-nodes in the CRT0066101-related network, we observed that the phosphorylation levels of YAP, AKT, and CDC2 were significantly decreased in the 


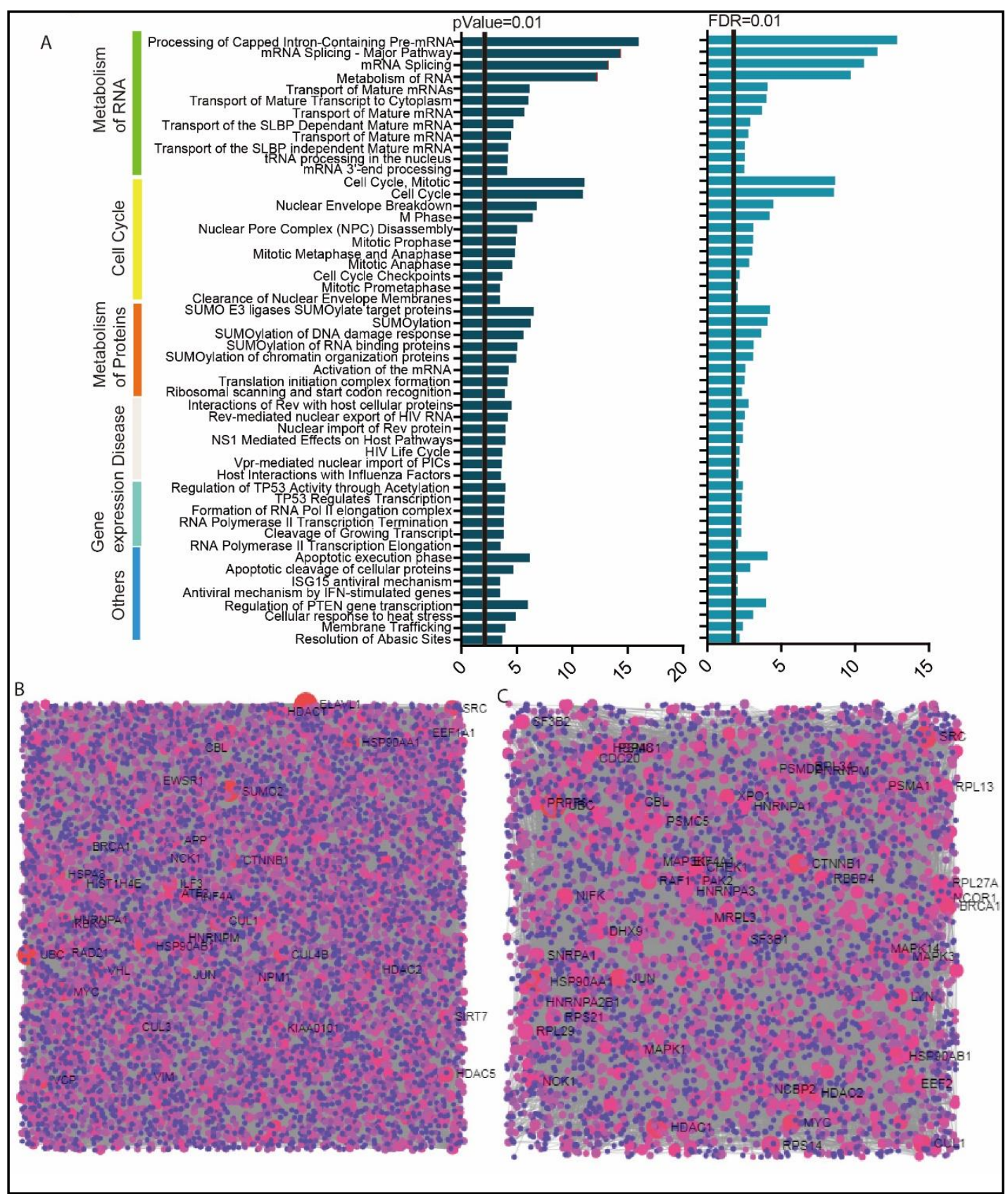

Fig. 5. Reactome and network analysis of PRKD-regulated phosphoproteins. (A) Enriched pathway analysis of PRKD-regulated phosphoproteins using the reactome. (B) Network analysis using the IMEx interactome database of CRT0066101-related phosphoproteins with labeled hub-nodes. (C) CRT0066101-related phosphoprotein network analyzed using the STRING interactome database with labeled hub-nodes.

CRT0066101-related table (Supplementary Table 5). It has been reported that CRT0066101 inhibits the phosphorylation level of YAP, AKT, and CDC2 in multiple cancer types [35, $38,39]$. MYC, MAPK1/3, AKT, and YAP play important roles in cancer progression. MYC is overexpressed in $30-50 \%$ of high-grade breast cancer $[40,41]$. In addition, MYC plays critical roles in multiple cellular pathways that promote cell survival and proliferation [42, 43]. MAPK1 and MAPK3 are major determinants in the control of diverse cellular processes such as tumor growth and survival [44]. AKT and YAP promote tumorigenesis via several oncogenic events such as cell apoptosis and cell growth [45, 46]. Cyclin-dependent kinases 


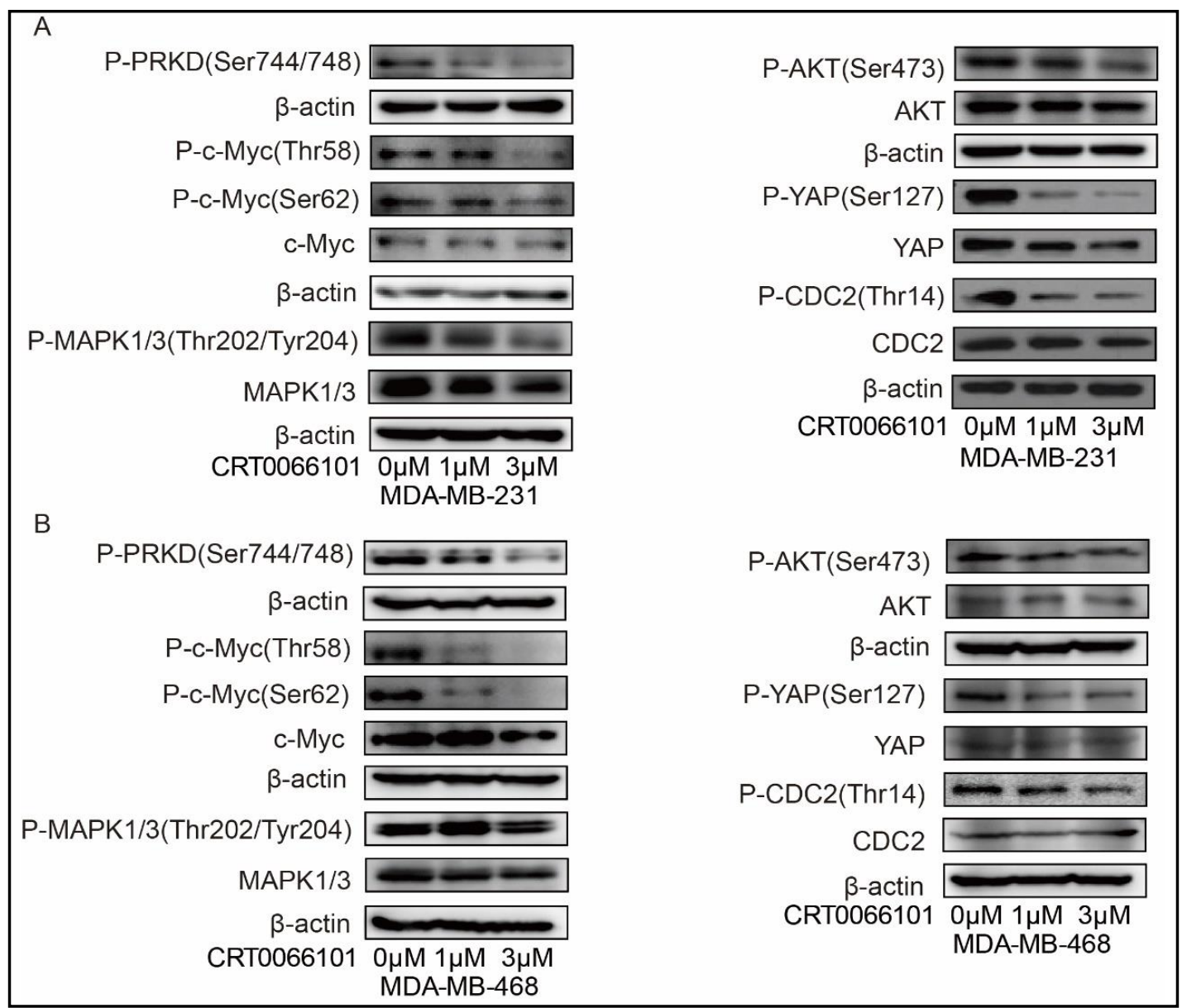

Fig. 6. Effect of CRT0066101 on the protein and phosphorylation levels of key regulators in TNBC. (A) MDA-MB-231 and (B) MDA-MB-468 cell lines were exposed to increasing concentrations (0, 1, and $3 \mu \mathrm{M})$ of CRT0066101 for 1 day. The protein and phosphorylation levels of PRKD, MYC, MAPK1/3, AKT, YAP, and CDC2 were examined by western blot.

(CDKs) regulate the cell cycle at several checkpoints, including G1 and G2/M [47]. The G1 checkpoint determines whether cells progress into $S$ phase, and CDC2 plays a role in G1/S progression.

To confirm that CRT0066101 regulates several targets in TNBC cells, we examined MYC, MAPK1/3, AKT, YAP, and CDC2 in selected model TNBC cell lines that were exposed to increasing concentrations $(0,1$, and $3 \mu \mathrm{M})$ of CRT0066101 for 1 day. The results show that CRT0066101 treatment decreased p-MYC, p-MAPK1/3, p-AKT, p-YAP, and p-CDC2 levels (Fig. 6). Similar results were also found by combined treatment with small interfering RNAs (siRNAs) against PRKD2 and PRKD3 (siPRKD2 and siPRKD3) (Supplementary Fig. S3).

\section{Discussion}

PRKDs are involved in a series of cancer-related processes including cell proliferation, apoptosis, differentiation, and cell cycle. Abnormal expression of PRKDs has been reported in various types of cancers, making them promising drug targets for cancer. In the present study, our results show that PRKD2 and PRKD3 are the two major PRKD isoforms expressed in breast cancer (Fig. 1). We also show that the PRKD pan-inhibitor CRT0066101 decreased TNBC cell proliferation in vitro and in vivo (Fig. 2 and 3), suggesting that CRT0066101 has 
antitumor activity in TNBC. These results are in accord with the effects of CRT0066101 reported in many other cancers including colon and pancreatic cancers [33, 35]. In addition, our study revealed that CRT0066101 treatment induced TNBC cell arrest at the G1 phase of the cell cycle and increased TNBC cell apoptosis (Fig. 2). Taken together, these results suggest that CRT0066101's inhibition of TNBC proliferation is mediated via induction of G1phase arrest and increasing the proportion of apoptotic cells.

Recently, several PRKD inhibitors, including CRT0066101, have been identified. Harikumar and colleagues found that CRT0066101 given orally significantly inhibits pancreatic tumor growth in vivo [33]. Wei and colleagues showed that CRT0066101 given orally suppresses colorectal cancer development in vivo [35]. Meanwhile, Borges et al. [27] demonstrated that CRT0066101 effectively targets estrogen-receptor-negative breast cancers. In our study, we show that CRT0066101 potently inhibited TNBC growth in vivo (Fig. 3). Our results, together with results from other groups, strongly indicate that CRT0066101 is an effective drug for the treatment of TNBC.

Although many reports have shown that CRT0066101 is an effective therapeutic in many different cancers, the mechanisms underlying this compound's anticancer effects are unknown. Since PRKDs are serine/threonine kinases, phosphoproteomic analysis was used to uncover the mechanism of CRT0066101's effects in breast cancer. Through reactome and network analyses, we found multiple hub-nodes related to cell proliferation, apoptosis, and the cell cycle.

In our study, we observed that the PRKD inhibitor CRT0066101 decreased breast cancer growth in vitro and in vivo, and increased breast cancer cell apoptosis and accumulation of cells in G1 phase. MYC plays key roles in cell growth [43], cell cycle [48], and apoptosis [49], and its aberrant expression has been shown to be involved in nearly all human cancers [43]. Our study showed that CRT0066101 significantly suppressed the phosphorylation of MYC on Thr58 and Ser62 in the investigated breast cancer cell lines. These sites are well documented to regulate MYC stability. Phosphorylation at Ser62 is known to stabilize c-MYC, whereas phosphorylation at Thr58 and Ser62 leads to its degradation by the proteasome [50, 51]. Our results show that when CRT0066101 or siPRKD2\&3 suppressed the phosphorylation of MYC on Thr58 and Ser62 in MDA-MB-468 cells, the level of MYC protein significantly decreased (Fig. 6B and Supplementary Fig. S3B). MYC protein levels were slightly decreased by CRT0066101 or siPRKD2\&3 in MDA-MB-231 cells (Fig. 6A and Supplementary Fig. S3A). These results were consistent with recently work reported by Xiaoxin Sun [52]. The decreased MYC protein level might due to other regulation mechanisms, for example, transcription repression upon treatment of CRT0066101 or siPRKD2\&3. The detail mechanism is worthy for further exploration. Meanwhile, PRKD inhibition restrained the MAPK1/3 and AKT signaling pathways. PRKD has been reported to regulate the MAPK pathway to promote cell growth [53]. In the current study, our results showed that CRT0066101 strongly decreased the phosphorylation of MAPK1/3 on Thr202 and Tyr204 and phosphor-AKT on Ser473. Taken together, these results show that the PRKD inhibitor CRT0066101 inhibits breast cancer progression via inhibition of the phosphorylation of MAPK1/3 and AKT. The HippoYAP pathway has been reported to mediate cell proliferation [54]. Our results show that PRKD inhibition led to a decrease in phosphorylation of YAP on Ser127. A related report showed that CRT0066101 prevented an increase in YAP phosphorylation on Ser127, YAP cytoplasmic accumulation, and increase in the mRNA levels of YAP/TEAD-regulated genes in intestinal epithelial cells. Furthermore, siRNA-mediated knockdown of PRKDs markedly attenuated YAP nuclear-cytoplasmic shuttling and phosphorylation at Ser127 $[39,55]$.

Cell cycle checkpoint loss is a hallmark of cancer. CDKs regulate the cell cycle at several checkpoints, including G1 and G2/M [47]. The G1 checkpoint determines whether cells go into $S$ phase, and CDC2 plays a role in G1/S procession. In our study, the level of phosphor-CDC2 (Thr14) was decreased, whereas the level of total CDC2 did not change in the CRT0066101-treated breast cancer cells. These results suggest that the G1 arrest by CRT0066101 in breast cancer cells is due to suppression of the phosphorylation of CDC2 on 
Thr14. This phosphorylation change was also confirmed using siPRKD2 and siPRKD3 (Supplementary Fig. S3).

\section{Conclusion}

Taken together, the results of the current study analyzing the phosphoproteome show that CRT0066101 is an effective drug for inhibiting proliferation, apoptosis, and cell cycle of TNBC via decreasing phosphorylation of MYC, MAPK1/3, AKT, YAP, and CDC2. The molecular mechanism underlying CRT0066101's effects in breast cancer is depicted schematically in Fig. 7, and provides new insights into the functions of PRKDs and supports the use of CRT0066101 for combating TNBC.

\section{Acknowledgements}

This work was funded by the

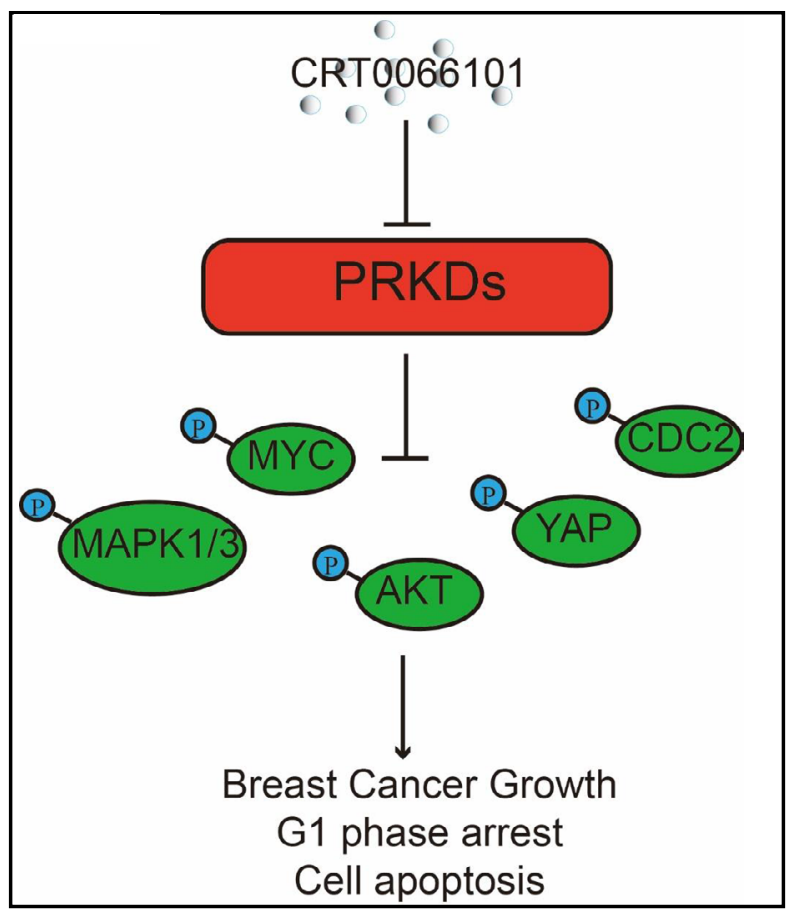

Fig. 7. Potential mechanism underlying how the PRKD inhibitor CRT0066101 decreases breast cancer growth via blocking cell cycle progression at the G1 phase and increasing cell apoptosis.

National Natural Science Foundation of China (Grant No. 81772956 \& 81572712 to L. Chen), the Key Research and Development Program of Jiangsu Province (Grant No. BE2017669 to J. Zhang), the National Basic Research Program of China (973 Program) (Grant No. 2015CB965000 to L. Chen), the Fundamental Research Funds for the Central Universities (Grant No. 3231005410 to L. Chen), the Natural Science Foundation of Jiangsu Province (Grant No. SBK2016030027 to L. Chen), the Six Talent Peaks Project in Jiangsu Province (Grant No. 2015-JY-002 to L. Chen), the Jiangsu Shuangchuang Talent Program (to L. Chen), the Jiangsu Province Post-Doctoral Fund (to Y. Liu), and the Science Foundation of the Chinese Academy of Sciences (Grant No. Y852126105 to Y. Liu).

\section{Disclosure Statement}

The authors declare that they have no conflict of interests.

\section{References}

1 Schneider BP, Winer EP, Foulkes WD, Garber J, Perou CM, Richardson A, Sledge GW, Carey LA: TripleNegative Breast Cancer: Risk Factors to Potential Targets. Clin Cancer Res 2008;14:8010-8018.

2 Dent R, Trudeau M, Pritchard KI, Hanna WM, Kahn HK, Sawka CA, Lickley LA, Rawlinson E, Sun P, Narod SA: Triple-negative breast cancer: Clinical features and patterns of recurrence. Clin Cancer Res 2007;13:44294434.

3 Irshad S, Ellis P, Tutt A: Molecular heterogeneity of triple-negative breast cancer and its clinical implications. Curr Opin Oncol 2011;23:566-577. 


\section{Cellular Physiology Cell Physiol Biochem 2019;52:382-396 \\ \begin{tabular}{c|l}
\hline DOI: 10.33594/000000027 & (c) 2019 The Author(s). Published by
\end{tabular} \\ and Biochemistry Published online: 5 March 2019 Cell Physiol Biochem Press GmbH\&Co. KG}

Liu et al.: Quantitative Phosphoproteomics Reveals Mechanism of CRT0066101

- 4 Karihtala P, Auvinen P, Kauppila S, Haapasaari KM, Jukkola-Vuorinen A, Soini Y: Vimentin, zeb1 and Sip1 are up-regulated in triple-negative and basal-like breast cancers: association with an aggressive tumour phenotype. Breast Cancer Res Treat 2013;138:81-90.

- 5 Sarrio D, Rodriguez-Pinilla SM, Hardisson D, Cano A, Moreno-Bueno G, Palacios J: Epithelial-mesenchymal transition in breast cancer relates to the basal-like phenotype. Cancer Res 2008;68:989-997.

- 6 Carey LA: Directed Therapy of Subtypes of Triple-Negative Breast Cancer. Oncologist 2011;16:71-78.

7 Perou CM: Molecular Stratification of Triple-Negative Breast Cancers. Oncologist 2011;16:61-70.

- 8 Zhukova E, Sinnett-Smith J, Rozengurt E: Protein kinase D potentiates DNA synthesis and cell proliferation induced by bombesin, vasopressin, or phorbol esters in Swiss 3T3 cells. J Biol Chem 2001;276:4029840305.

- 9 Wong C, Jin ZG: Protein kinase C-dependent protein kinase D activation modulates ERK signal pathway and endothelial cell proliferation by vascular endothelial growth factor. J Biol Chem 2005;280:33262-33269.

10 Eiseler T, Doppler H, Yan IK, Goodison S, Storz P: Protein kinase D1 regulates matrix metalloproteinase expression and inhibits breast cancer cell invasion. Breast Cancer Res 2009;11:R13.

11 Peterburs P, Heering J, Link G, Pfizenmaier K, Olayioye MA, Hausser A: Protein kinase D regulates cell migration by direct phosphorylation of the cofilin phosphatase slingshot 1 like. Cancer Res 2009;69:56345638.

- 12 Du C, Zhang C, Hassan S, Biswas MH, Balaji KC: Protein kinase D1 suppresses epithelial-to-mesenchymal transition through phosphorylation of snail. Cancer Res 2010;70:7810-7819.

- 13 Qin L, Zeng H, Zhao D: Requirement of protein kinase D tyrosine phosphorylation for VEGF-A165-induced angiogenesis through its interaction and regulation of phospholipase Cgamma phosphorylation. J Biol Chem 2006;281:32550-32558.

- 14 Hao Q, McKenzie R, Gan H, Tang H: Protein kinases D2 and D3 are novel growth regulators in HCC1806 triple-negative breast cancer cells. Anticancer Res 2013;33:393-399.

- 15 Sharlow ER, Giridhar KV, LaValle CR, Chen J, Leimgruber S, Barrett R, Bravo-Altamirano K, Wipf P, Lazo JS, Wang QJ: Potent and selective disruption of protein kinase D functionality by a benzoxoloazepinolone. J Biol Chem 2008;283:33516-33526.

16 Sundram V, Ganju A, Hughes JE, Khan S, Chauhan SC, Jaggi M: Protein kinase D1 attenuates tumorigenesis in colon cancer by modulating beta-catenin/T cell factor activity. Oncotarget 2014;5:6867-6884.

17 Zou Z, Zeng F, Xu W, Wang C, Ke Z, Wang QJ, Deng F: PKD2 and PKD3 promote prostate cancer cell invasion by modulating NF-kappaB- and HDAC1-mediated expression and activation of uPA. J Cell Sci 2012;125:4800-4811.

- 18 Azoitei N, Pusapati GV, Kleger A, Moller P, Kufer R, Genze F, Wagner M, van Lint J, Carmeliet P, Adler G, Seufferlein T: Protein kinase D2 is a crucial regulator of tumour cell-endothelial cell communication in gastrointestinal tumours. Gut 2010;59:1316-1330.

- 19 Kim M, Jang HR, Kim JH, Noh SM, Song KS, Cho JS, Jeong HY, Norman JC, Caswell PT, Kang GH, Kim SY, Yoo HS, Kim YS: Epigenetic inactivation of protein kinase D1 in gastric cancer and its role in gastric cancer cell migration and invasion. Carcinogenesis 2008;29:629-637.

- 20 Steffensen AB, Bomholtz SH, Andersen MN, Olsen JV, Mutsaers N, Lundegaard PR, Lundby A, Schmitt N: PKD Phosphorylation as Novel Pathway of K(V)11.1 Regulation. Cell Physiol Biochem 2018;47:1742-1750.

21 Karam M, Legay C, Auclair C, Ricort JM: Protein kinase D1 stimulates proliferation and enhances tumorigenesis of MCF-7 human breast cancer cells through a MEK/ERK-dependent signaling pathway. Exp Cell Res 2012;318:558-569.

22 Borges S, Doppler H, Perez EA, Andorfer CA, Sun Z, Anastasiadis PZ, Thompson E, Geiger XJ, Storz P: Pharmacologic reversion of epigenetic silencing of the PRKD1 promoter blocks breast tumor cell invasion and metastasis. Breast Cancer Res 2013;15:R66.

23 Eiseler T, Doppler H, Yan IK, Kitatani K, Mizuno K, Storz P: Protein kinase D1 regulates cofilin-mediated F-actin reorganization and cell motility through slingshot. Nat Cell Biol 2009;11:545-556.

- 24 Huck B, Duss S, Hausser A, Olayioye MA: Elevated protein kinase D3 (PKD3) expression supports proliferation of triple-negative breast cancer cells and contributes to mTORC1-S6K1 pathway activation. J Biol Chem 2014;289:3138-3147.

- 25 Chen J, Lu L, Feng Y, Wang H, Dai L, Li Y, Zhang P: PKD2 mediates multi-drug resistance in breast cancer cells through modulation of P-glycoprotein expression. Cancer Lett 2011;300:48-56. 


\section{Cellular Physiology Cell Physiol Biochem 2019;52:382-396 \\ \begin{tabular}{c|c|c|}
\hline DOl: 10.33594/000000027 & (c) 2019 The Author(s). Published by
\end{tabular} \\ and Biochemistry Published online: 5 March 2019 Cell Physiol Biochem Press GmbH\&Co. KG}

Liu et al.: Quantitative Phosphoproteomics Reveals Mechanism of CRT0066101

26 Borges S, Doppler HR, Storz P: A combination treatment with DNA methyltransferase inhibitors and suramin decreases invasiveness of breast cancer cells. Breast Cancer Res Treat 2014;144:79-91.

27 Borges S, Perez EA, Thompson EA, Radisky DC, Geiger XJ, Storz P: Effective Targeting of Estrogen ReceptorNegative Breast Cancers with the Protein Kinase D Inhibitor CRT0066101. Mol Cancer Ther 2015;14:13061316.

28 Liu Y, Li J, Zhang J, Yu Z, Yu S, Wu L, Wang Y, Gong X, Wu C, Cai X, Mo L, Wang M, Gu J, Chen L: Oncogenic Protein Kinase D3 Regulating Networks in Invasive Breast Cancer. Int J Biol Sci 2017; 13:748-758.

29 George KM, Frantz MC, Bravo-Altamirano K, Lavalle CR, Tandon M, Leimgruber S, Sharlow ER, Lazo JS, Wang QJ, Wipf P: Design, Synthesis, and Biological Evaluation of PKD Inhibitors. Pharmaceutics 2011;3:186-228.

30 Gamber GG, Meredith E, Zhu Q Yan W, Rao C, Capparelli M, Burgis R, Enyedy I, Zhang JH, Soldermann N, Beattie K, Rozhitskaya O, Koch KA, Pagratis N, Hosagrahara V, Vega RB, McKinsey TA, Monovich L: 3,5-diarylazoles as novel and selective inhibitors of protein kinase D. Bioorg Med Chem Lett 2011;21:14471451.

31 Meredith EL, Ardayfio O, Beattie K, Dobler MR, Enyedy I, Gaul C, Hosagrahara V, Jewell C, Koch K, Lee W, Lehmann H, McKinsey TA, Miranda K, Pagratis N, Pancost M, Patnaik A, Phan D, Plato C, Qian M, Rajaraman V, et al.: Identification of orally available naphthyridine protein kinase D inhibitors. J Med Chem 2010;53:5400-5421.

32 Evans IM, Bagherzadeh A, Charles M, Raynham T, Ireson C, Boakes A, Kelland L, Zachary IC: Characterization of the biological effects of a novel protein kinase D inhibitor in endothelial cells. Biochem J 2010;429:565-572.

33 Harikumar KB, Kunnumakkara AB, Ochi N, Tong Z, Deorukhkar A, Sung B, Kelland L, Jamieson S, Sutherland R, Raynham T, Charles M, Bagherzadeh A, Foxton C, Boakes A, Farooq M, Maru D, Diagaradjane P, Matsuo Y, Sinnett-Smith J, Gelovani J, et al.: A novel small-molecule inhibitor of protein kinase D blocks pancreatic cancer growth in vitro and in vivo. Mol Cancer Ther 2010;9:1136-1146.

34 Lavalle CR, Bravo-Altamirano K, Giridhar KV, Chen J, Sharlow E, Lazo JS, Wipf P, Wang QJ: Novel protein kinase D inhibitors cause potent arrest in prostate cancer cell growth and motility. BMC Chem Biol 2010;10:5.

35 Wei N, Chu E, Wipf P, Schmitz JC: Protein kinase d as a potential chemotherapeutic target for colorectal cancer. Mol Cancer Ther 2014;13:1130-1141.

36 Neve RM, Chin K, Fridlyand J, Yeh J, Baehner FL, Fevr T, Clark L, Bayani N, Coppe JP, Tong F, Speed T, Spellman PT, DeVries S, Lapuk A, Wang NJ, Kuo WL, Stilwell JL, Pinkel D, Albertson DG, Waldman FM, et al.: A collection of breast cancer cell lines for the study of functionally distinct cancer subtypes. Cancer Cell 2006;10:515-527.

- 37 Stein LD: Using the Reactome database. Curr Protoc Bioinformatics 2004;Chapter 8:Unit 8.7.

- 38 Li QQ, Hsu I, Sanford T, Railkar R, Balaji N, Sourbier C, Vocke C, Balaji KC, Agarwal PK: Protein kinase D inhibitor CRT0066101 suppresses bladder cancer growth in vitro and xenografts via blockade of the cell cycle at G2/M. Cell Mol Life Sci 2018;75:939-963.

39 Wang J, Sinnett-Smith J, Stevens JV, Young SH, Rozengurt E: Biphasic Regulation of Yes-associated Protein (YAP) Cellular Localization, Phosphorylation, and Activity by G Protein-coupled Receptor Agonists in Intestinal Epithelial Cells: A NOVEL ROLE FOR PROTEIN KINASE D (PKD). J Biol Chem 2016;291:1798818005.

40 Deming SL, Nass SJ, Dickson RB, Trock BJ: C-myc amplification in breast cancer: a meta-analysis of its occurrence and prognostic relevance. Br J Cancer 2000;83:1688-1695.

41 Blancato J, Singh B, Liu A, Liao DJ, Dickson RB: Correlation of amplification and overexpression of the c-myc oncogene in high-grade breast cancer: FISH, in situ hybridisation and immunohistochemical analyses. $\mathrm{Br} \mathrm{J}$ Cancer 2004;90:1612-1619.

- 42 Meyer N, Penn LZ: Reflecting on 25 years with MYC. Nat Rev Cancer 2008;8:976-990.

- 43 Dang CV: MYC on the path to cancer. Cell 2012;149:22-35.

- 44 Kohno M, Pouyssegur J: Targeting the ERK signaling pathway in cancer therapy. Ann Med 2006;38:200-211.

- 45 Vivanco I, Sawyers CL: The phosphatidylinositol 3-Kinase AKT pathway in human cancer. Nat Rev Cancer 2002;2:489-501.

46 Harvey KF, Zhang X, Thomas DM: The Hippo pathway and human cancer. Nat Rev Cancer 2013;13:246-257. 


\section{Cellular Physiology and Biochemistry}

Cell Physiol Biochem 2019;52:382-396

\begin{tabular}{l|l}
\hline DOI: 10.33594/000000027 & (c) 2019 The Author(s). Published by
\end{tabular}

\begin{tabular}{l|l} 
Liu et al.: Quantitative Phosphoproteomics Reveals Mechanism of CRT0066101 \\
\hline
\end{tabular}

47 Qiao L, Zheng J, Tian Y, Zhang Q, Wang X, Chen JJ, Zhang W: Regulator of chromatin condensation 1 abrogates the G1 cell cycle checkpoint via Cdk1 in human papillomavirus E7-expressing epithelium and cervical cancer cells. Cell Death Dis 2018;9:583.

48 Rabbitts PH, Watson JV, Lamond A, Forster A, Stinson MA, Evan G, Fischer W, Atherton E, Sheppard R, Rabbitts TH: Metabolism of c-myc gene products: c-myc mRNA and protein expression in the cell cycle. EMBO J 1985;4:2009-2015.

49 Okuyama H, Endo H, Akashika T, Kato K, Inoue M: Downregulation of c-MYC protein levels contributes to cancer cell survival under dual deficiency of oxygen and glucose. Cancer Res 2010;70:10213-10223.

50 Hann SR: Role of post-translational modifications in regulating c-Myc proteolysis, transcriptional activity and biological function. Semin Cancer Biol 2006;16:288-302.

51 Vervoorts J, Luscher-Firzlaff J, Luscher B: The ins and outs of MYC regulation by posttranslational mechanisms. J Biol Chem 2006;281:34725-34729.

52 Sun XX, Chen YX, Su YL, Wang XY, Chauhan KM, Liang J, Daniel CJ, Sears RC, Dai MS: SUMO protease SENP1 deSUMOylates and stabilizes c-Myc. P Natl Acad Sci USA 2018;115:10983-10988.

53 Sinnett-Smith J, Zhukova E, Hsieh N, Jiang X, Rozengurt E: Protein kinase D potentiates DNA synthesis induced by Gq-coupled receptors by increasing the duration of ERK signaling in swiss 3T3 cells. J Biol Chem 2004;279:16883-16893.

54 Gumbiner BM, Kim NG: The Hippo-YAP signaling pathway and contact inhibition of growth. J Cell Sci 2014;127:709-717.

- 55 Hao F, Xu QH, Zhao YL, Stevens JV, Young SH, Sinnett-Smith J, Rozengurt E: Insulin Receptor and GPCR Crosstalk Stimulates YAP via PI3K and PKD in Pancreatic Cancer Cells. Mol Cancer Res 2017;15:929-941. 